\title{
气液界面胶体球刻蚀法制备二维有序多孔薄膜
}

\author{
李扬齐利民* \\ (北京分子科学国家实验室 分子动态与稳态国家重点实验室 北京大学化学与分子工程学院 北京 100871)
}

\begin{abstract}
摘要 作为一类重要的二维材料, 二维有序多孔薄膜受到人们的广泛关注. 气液界面胶体球刻蚀法是近些年发展起来 的一种以漂浮在液面上的单层胶体晶体为模板来制备二维有序纳米结构的方法，具有简单、高效、重现性好、适用范 围广以及结构参数易调变等优点. 近年来, 我们课题组利用气液界面胶体球刻蚀法实现了包括多种无机物纳米网、纳 米碗阵列和纳米网-纳米碗复合阵列在内的一系列自支撑二维有序多孔薄膜的可控制备, 考察了其二维光子晶体性质, 并研究了其在刻蚀掩膜、溶剂检测、生物传感、电阻开关器件、光电化学分解水等方面的应用. 本文在重点介绍我们 课题组研究进展的同时，也简要总结了该领域的整体发展状况并展望了该领域的今后发展方向.
\end{abstract}

关键词 单层胶体晶体; 胶体球刻蚀法; 气液界面; 二维有序纳米结构; 自支撑多孔薄膜; 纳米网; 纳米碗阵列

\section{Advances in Fabrication of Two-dimensionally Ordered Porous Membranes by Nanosphere Lithography at the Gas-liquid Interface}

\author{
Li, Yang Qi, Limin* \\ (Beijing National Laboratory for Molecular Sciences, State Key Laboratory for Structural Chemistry of Unstable and Stable \\ Species, College of Chemistry and Molecular Engineering, Peking University, Beijing 100871)
}

\begin{abstract}
Two-dimensionally (2D) ordered porous membranes are attractive structures with potential applications in a broad variety of fields such as separation and purification, controlled drug delivery, scaffolds for tissue engineering, biosensors, wetting and adhesion, photonic crystals and optical devices, electric and optoelectric devices, antireflective coatings, low dielectric constant materials, and templates or microreactors for nanofabrication. Nanosphere lithography (NSL) based on monolayer colloidal crystals (MCCs) is a general nanofabrication approach toward 2D patterned nanostructures with high controllability and reproducibility. Recently, nanosphere lithography at the gas-liquid interface, which employs MCCs floating at the solution surface as templates, has been demonstrated to be a facile, inexpensive, efficient, and versatile method for the controllable fabrication of self-standing, large-area, 2D ordered porous membranes with tunable structural parameters. This account summarizes our recent efforts devoted to the fabrication and applications of a variety of inorganic $2 \mathrm{D}$ ordered porous membranes including nanonets, nanobowl arrays and nanonet-nanobowl composite arrays by NSL at the gas-liquid interface via controlled chemical deposition. First, free-standing, hexagonally ordered $\mathrm{Ag}_{2} \mathrm{~S}$ nanonets were prepared through interfacial deposition induced by gas diffusion; moreover, a variety of $2 \mathrm{D}$ ordered gold nanoarrays with unusual patterns were produced by using nanonet bilayers as unique deposition masks. Second, free-standing, honeycomb-patterned nanobowl arrays of $\mathrm{Ag}$ and $\mathrm{CaCO}_{3}$ were fabricated through gas diffusion-induced deposition whereas large-area $\mathrm{ZnS}$ nanobowl arrays with high regularity were produced through direct solution deposition. The potential applications of the obtained Ag and $\mathrm{ZnS}$ nanobowl arrays as plasmonic crystal-based and photonic crystal-based sensors were demonstrated, respectively. Third, unique $\mathrm{Ag}_{2} \mathrm{~S}-\mathrm{Ag}$ heterostructured nanobowl arrays were fabricated by two-step NSL at the gas-liquid interface, which involved the successive deposition of $\mathrm{Ag}_{2} \mathrm{~S}$ nanonets and $\mathrm{Ag}$ nanobowl arrays. The produced $\mathrm{Ag}_{2} \mathrm{~S}-\mathrm{Ag}$ heterostructured nanobowl arrays exhibited effective resistance switching behaviors and enhanced photoresponses, showing potential applications in both electric devices and photocatalysis. Finally, the research trend in this field is briefly described and the remaining challenges are discussed.
\end{abstract}

Keywords monolayer colloidal crystals; nanosphere lithography; gas-liquid interface; 2D ordered nanostructures; self-standing porous membranes; nanonets; nanobowl arrays

\section{1 引言}

二维有序纳米结构往往呈现出有序图案化结构所 带来的新颖性质或优化性能, 在光学器件、电学器件、
能源转换与存储、生物和化学传感、分离与控释、表面 浸润与粘附等领域有着广阔的应用前景 ${ }^{[1 \sim 6]}$. 因此, 人 们多年来致力于发展可高效制备结构参数可调的大面 积二维有序纳米结构的表面纳米图案化技术. 相比于传

* E-mail: liminqi@pku.edu.cn

Received February 28, 2015; published April 27, 2015.

Project supported by the National Natural Science Foundation of China (Nos. 21173010 and 21473004) and the National Key Basic Research Program of China (No. 2013CB932601).

项目受国家自然科学基金(Nos. 21173010, 21473004)和国家重点基础研究发展计划(No. 2013CB932601)资助. 
统的刻蚀技术(如光刻、电子束刻蚀、X-射线刻蚀等)和 近年来发展起来的纳米压印、沾笔刻蚀、微接触印刷等 技术，基于自组装模板的表面纳米图案化技术因其省 时、低成本、高产量等特点而受到广泛关注 ${ }^{[7]}$. 作为一 种典型的二维结构化的自组装模板, 单层胶体晶体 (MCC) 被广泛用于各类二维有序纳米结构的可控制备. 这种以单层胶体晶体作为模板进行蚀刻或沉积的二维 纳米图案化技术通常被称作为胶体球刻蚀法(或纳米球 刻蚀法), 它具有简单、高效、重现性好以及结构参数易 调变等优点, 因而日益受到人们的重视 ${ }^{[8 \sim 13]}$. 作为二维 模板的单层胶体晶体可以附着于固体表面进行气固界 面沉积或液固界面沉积, 从而得到紧密附着于固体表面 上的二维有序纳米结构; 另一方面, 单层胶体晶体可以 漂浮于液体表面进行气液界面沉积, 从而得到漂浮于液 体表面的自支撑二维有序多孔薄膜, 进而可转移至任意 基底表面. 因此, 基于气液界面沉积过程的气液界面胶 体球刻蚀法在制备自支撑的大面积二维有序多孔薄膜 方面具有显著优势.

二维有序多孔薄膜是一类重要的二维有序纳米结 构, 它在分离与提纯、药物控释、生物组织工程支架、 生物传感、浸润与粘附、光子晶体与光学器件、电学与 光电器件、减反射涂层、低介电常数薄膜以及可用于纳 米制备的模板或微反应器等领域有着诸多实际应用和 潜在应用 ${ }^{[2 \sim 4]}$. 一方面, 这种薄膜具有高度有序的周期 性网孔结构, 是一种典型的二维光子晶体材料 ${ }^{[1]}$. 它往 往呈现出明亮的结构色, 并由于对周围环境的敏感性而 可应用于传感检测. 另一方面, 这种薄膜具有尺度均一 且形貌规整的网孔, 因而被看作可应用于物质选择性输 运和分离、蛋白质及药物的长期可控释放、酶固定及生 物传感等领域的理想薄膜材料 ${ }^{[2]}$. 模板法在二维有序多 孔薄膜的可控制备中发挥着重要作用, 而漂浮于液体表 面上的自组装模板对于快速制备自支撑二维有序多孔 薄膜尤为有利. 基于液体表面水滴模板的呼吸图案法 $[14,15]$ 和基于液体表面胶体球模板的气液界面胶体球刻蚀法 ${ }^{[1]}$ 是其中的两个典型代表. 呼吸图案法非常适合于制备聚 合物有序多孔薄膜, 但适用的物质种类范围较为有限, 并且其内在机理仍不清晰, 尚难于实现大面积无缺陷的 有序多孔薄膜的可控制备 ${ }^{[15]}$. 气液界面胶体球刻蚀法 则可以适用于从聚合物到无机物的多种类物质, 并且合 成机理简单明了, 易于可控制备大面积规整的二维有序 多孔薄膜. 我们课题组最近在胶体晶体模板合成方面开 展了一系列研究, 下文主要介绍我们利用气液界面胶体 球刻蚀法制备二维有序多孔薄膜的研究进展.

\section{2 气液界面胶体球刻蚀法基本原理}

气液界面胶体球刻蚀法主要以漂浮在液体表面的 单层胶体晶体为模板, 通过气液界面附近的化学反应 (如聚合或沉淀反应), 复制得到目标产物的自支撑二维
有序纳米结构. 该方法最初被应用于聚合物有序多孔薄 膜的可控制备. 2003 年, Goedel 等 ${ }^{[16]}$ 首次利用该方法成 功制备得到了聚合物的网状自支撑多孔薄膜. 先将聚合 物单体和二氧化硅胶球的混合液铺展在水面上，可以得 到一层聚合物单体填充的单层胶体晶体薄膜; 然后向体 系中加入引发剂便可发生聚合反应，即可得到胶体晶体一 聚合物复合薄膜. 该复合薄膜可转移到任意的基底上, 除去胶球，便可得到孔径均一的聚合物网状薄膜. 通过 调节胶球直径, 可以得到孔径在 $20 \sim 100 \mathrm{~nm}$ 大范围可 调的聚合物网状薄膜 ${ }^{[17]}$. 这种网状薄膜可以用作模板 来制备金属 ${ }^{[18]}$ 和金属氧化物 ${ }^{[19]}$ 的环状颗粒. 若将聚合 物单体直接溶解在液相中, 聚合反应可以优先在胶球与 溶液的界面处进行，最终得到自支撑的聚合物纳米碗阵 列. Manohar 等 ${ }^{[20]}$ 利用此方法成功制备得到了聚苯胺纳 米碗阵列薄膜. 若以 $\mathrm{HAuCl}_{4}$ 溶液为氧化剂使苯胺单体 氧化聚合, 还可得到 $\mathrm{Au}$ 纳米粒子功能化的聚苯胺纳米 碗阵列, 它可作为对氨气快速响应的气体传感器 ${ }^{[21]}$.

上述早期工作所得到的都是聚合物多孔薄膜，如何 将气液界面胶体球刻蚀法扩展至二维有序无机物多孔 薄膜的可控制备则是人们面临的一个挑战. 值得一提的 是, Sun 等 ${ }^{[22]}$ 以漂浮在 $\mathrm{HAuCl}_{4}$ 溶液表面的单层胶体晶体 作为模板, 通过光化学反应制备了 $\mathrm{Au}$ 的纳米碗阵列结 构. 近些年, 我们课题组发展了一种利用气液界面胶球 刻蚀法生长自支撑的、大面积二维有序无机多孔薄膜的 通用合成方法, 可以分别实现无机纳米网、纳米碗阵列 和纳米网-纳米碗复合阵列的可控制备(图 1). 首先利用 气液界面自组装法制备得到由聚合物胶球六方密堆排 列而成的 $\mathrm{MCC}$, 然后将其转移到反应液表面, 进而通 过气体扩散或液相沉积在胶球表面沉积反应产物. 由于 胶球自身重力, 溶液表面通常略高于胶球的赤道面, 而 毛细作用则会导致三球之间缝隙处的液面上升, 形成略 高于正常液面高度的弯月面. 当化学反应导致的沉积发 生时, 既可能优先发生于胶球上部的气液界面处, 也可 能优先发生于胶球下部的液固界面处, 这将在很大程度 上取决于沉积物与胶球表面的亲和能力. 若反应产物优 先沉积于溶液表面(途径 1), 则可以得到纳米网与 MCC 的复合薄膜; 它可转移至任意的基底表面, 溶解除去 MCC 模板后即可得到自支撑的无机纳米网薄膜(A). 若 反应产物优先沉积于胶球下表面(途径 2), 则可以得到 纳米碗阵列与 $\mathrm{MCC}$ 的复合薄膜; 它也可转移至任意的 基底表面，溶解除去 MCC 模板后即可得到自支撑的无 机纳米碗阵列薄膜(B). 若通过两步化学沉积, 还可利 用气液界面胶体球刻蚀法得到纳米网一纳米碗复合阵列. 这可以通过两种不同的沉积次序来实现：先沉积某种物 质的纳米网(途径 1), 再沉积另一种物质的纳米碗(途径 3 ), 或者先沉积某种物质的纳米碗(途径 2), 再沉积另一 种物质的纳米网(途径 4). 两种方式均可得到纳米网、纳 米碗阵列与 MCC 的复合物. 在除去 MCC 模板后, 即可 
得到由上层纳米网与下层纳米碗阵列复合而成的纳米 网一纳米碗复合阵列 $(\mathrm{C})$, 它是一种结构新颖的异质结构 纳米碗阵列.

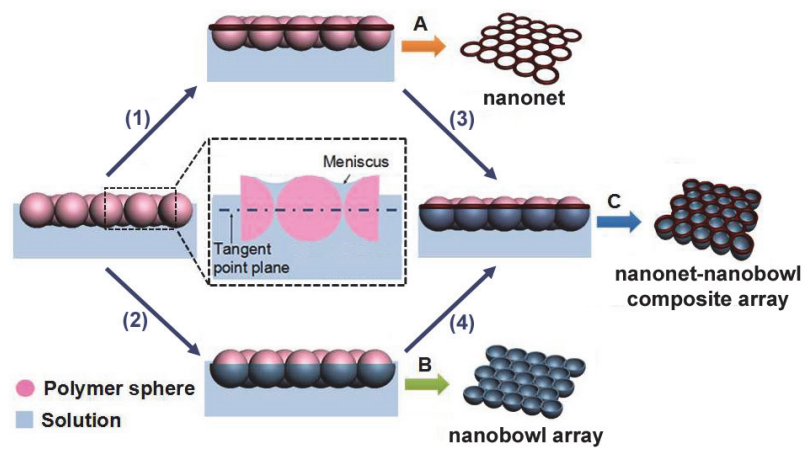

图 1 气液界面胶体球刻蚀法制备二维有序纳米结构过程示意图. (A) 纳米网; (B) 纳米碗阵列; (C) 纳米网-纳米碗复合阵列

Figure 1 Schematic illustration of the fabrication of $2 \mathrm{D}$ ordered nanostructures by nanosphere lithography at the gas-liquid interface: (A) nanonets, (B) nanobowl array, (C) nanonet-nanobowl composite array

\section{3 纳米网}

作为纳米网制备的典型实例，我们利用气液界面胶 体球刻蚀法并结合气体扩散反应制备了自支撑的 $\mathrm{Ag}_{2} \mathrm{~S}$ 纳米网薄膜(图 2) ${ }^{[23]}$. 以 $\mathrm{AgNO}_{3}$ 溶液为反应液, 由硫代 乙酰胺(TAA)缓慢释放出的 $\mathrm{H}_{2} \mathrm{~S}$ 气体逐渐扩散至反应液 处, 并在溶液表面发生 $\mathrm{Ag}_{2} \mathrm{~S}$ 的沉积(图 2a). 若以直径为 $500 \mathrm{~nm}$ 的聚苯乙烯(PS)胶球 MCC 为模板, 室温下反应 $48 \mathrm{~h}$, 可得到面积约为 $1 \mathrm{~cm}^{2}$ 的具有六方排列孔结构的 $\mathrm{Ag}_{2} \mathrm{~S}$ 网状薄膜, 网孔直径约 $400 \mathrm{~nm}$ 、网线宽约 100 $\mathrm{nm}$ (图 2b, 2c). 虽然该纳米网薄膜厚度很小 $(c a .100 \mathrm{~nm})$, 仍具有良好的自我支撑能力和机械强度, 并且具有一定 的柔性, 可以在一定程度上折叠变形(图 2d). 纳米网的 网孔尺寸可由使用具有不同胶球直径的 MCC 模板进行 有效调节. 随着反应时间的延长, 纳米网薄膜的厚度增 大、网孔缩小. 这种大面积规整有序的 $\mathrm{Ag}_{2} \mathrm{~S}$ 纳米网薄 膜由于网孔的周期性排列而具有二维光子晶体的特性, 在透射光谱中呈现出明显的波谷, 对应于二维光子晶体 的带隙(图 2e). 随着 MCC 胶球直径的增大, 纳米网的重 复周期增大, 透射波谷发生红移, 并且光子带隙波长与 胶球直径呈良好的线性关系.

由于该方法所制备的 $\mathrm{Ag}_{2} \mathrm{~S}$ 纳米网具有大面积连 续、网孔直径易调变、可转移到任意基底上等特点, 该 纳米网薄膜还可进一步作为制备其他图案化阵列结构 的掩膜. 如果将单层的纳米网薄膜转移到固体基底上, 作为掩膜来蒸镀一层 $\mathrm{Au}$ 膜, 除去纳米网后便可得到 $\mathrm{Au}$ 纳米盘阵列. 除了利用单层纳米网薄膜为掩膜, 若采用 两次捞膜得到的双层纳米网薄膜为掩膜, 则可构筑出更 加复杂的图案化纳米阵列结构. 如图 3 所示, 由于两层 纳米网的相对转角不同, 即网孔之间的相位不同, 两层 纳米网薄膜的叠加会产生多种图案. 利用这些图案为掩
膜来蒸镀 $\mathrm{Au}$ ，即可得到多种新颖奇特的 $\mathrm{Au}$ 纳米颗粒阵 列.

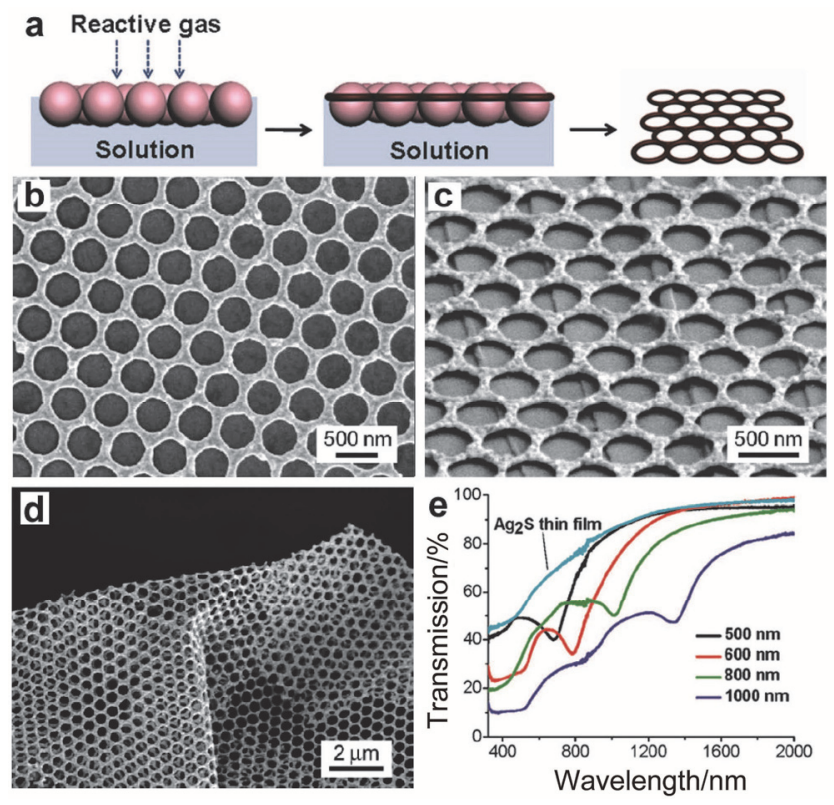

图 2 (a) 基于气体扩散的气液界面胶体球刻蚀法制备纳米网示意图; $(\mathrm{b} \sim \mathrm{d})$ 直径 $500 \mathrm{~nm}$ 胶球为模板制备的 $\mathrm{Ag}_{2} \mathrm{~S}$ 纳米网的 SEM 照片; (e) 不同直径胶球为模板制备的 $\mathrm{Ag}_{2} \mathrm{~S}$ 纳米网的透射光谱 ${ }^{[23]}$

Figure 2 (a) Schematic of the fabrication of nanonets by nanosphere lithography at the gas-liquid interface via gas diffusion. $(b \sim d)$ SEM images of $\mathrm{Ag}_{2} \mathrm{~S}$ nanonets prepared using colloidal spheres $c a .500 \mathrm{~nm}$ in diameter. (e) Transmission spectra of $\mathrm{Ag}_{2} \mathrm{~S}$ nanonets prepared using colloidal spheres with different particle diameters as well as unstructured $\mathrm{Ag}_{2} \mathrm{~S}$ thin films ${ }^{[23]}$

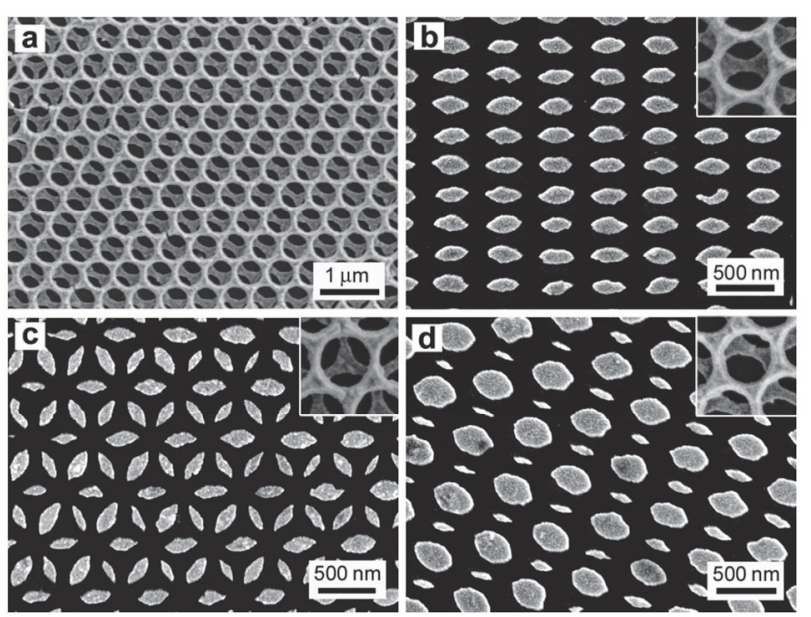

图 3 双层 $\mathrm{Ag}_{2} \mathrm{~S}$ 纳米网薄膜 $(\mathrm{a})$ 和以双层 $\mathrm{Ag}_{2} \mathrm{~S}$ 纳米网薄膜为掩膜制备 的 $\mathrm{Au}$ 纳米颗粒阵列 $(\mathrm{b} \sim \mathrm{d})$ 的 SEM 照片; 插图显示作为掩膜的具有不 同相对转角的双层 $\mathrm{Ag}_{2} \mathrm{~S}$ 纳米网 ${ }^{[23]}$

Figure 3 SEM images of a bilayer $\mathrm{Ag}_{2} \mathrm{~S}$ nanonet membrane (a) and gold nanopatterns created by evaporation deposition with bilayer $\mathrm{Ag}_{2} \mathrm{~S}$ nanonets as the deposition masks $(b \sim d)$. Insets show the corresponding bilayer nanonets with different phase shifts ${ }^{[23]}$

\section{4 纳米碗阵列}

气液界面胶体球刻蚀法适用于多种无机纳米碗阵 
列的可控制备. 例如, 我们利用气液界面胶体球刻蚀法 并结合气体扩散反应制备了大面积自支撑的 $\mathrm{Ag}$ 纳米碗 阵列薄膜(图 4) ${ }^{[24]}$. 以 $\mathrm{AgNO}_{3}$ 溶液为反应液, 以水合肼 和浓氨水的混合液为气体源向溶液中缓慢扩散 $\mathrm{N}_{2} \mathrm{H}_{4} / \mathrm{NH}_{3}$ 混合气体, 便会在胶球底部生长得到 $\mathrm{Ag}$ 纳米 碗阵列(图 4a). 六方密堆排列而成的纳米碗阵列中相邻 两个碗中心的间距为 $450 \mathrm{~nm}$, 与 MCC 模板中胶球的直 径相匹配(图 4b,4c), 说明纳米碗阵列完整的复制了 $\mathrm{MCC}$ 模板的周期性结构. 从图中可看出, 在三球空隙 处会有一个三角形的突起, 这是由于在 MCC 模板中三 球空隙处的溶液受毛细作用的影响而显著高于胶球赤 道面的缘故. 图 $4 \mathrm{~d}$ 显示该纳米碗阵列薄膜大面积连续, 且薄膜的表观厚度约为 $0.5 \mu \mathrm{m}$. 所得到的大面积规整有 序的 $\mathrm{Ag}$ 纳米碗阵列是一种典型的等离激元晶体薄膜, 其反射光谱在 $440 \mathrm{~nm}$ 有一个较锐的反射波谷, 并在 750 $\mathrm{nm}$ 存在一个较宽的反射波谷(图 4e). 鉴于等离激元晶 体具有显著的环境依赖性质, 该 $\mathrm{Ag}$ 纳米碗阵列薄膜在 小分子及生物大分子检测具有潜在的应用价值. 与此同 时, 这种 $\mathrm{Ag}$ 纳米碗阵列还可作为良好的表面增强 Raman 散射 (SERS) 基底来实现对于对氨基苯硫酚 (4-ATP)分子的检测. 类似的, 通过扩散入反应液的 $\mathrm{CO}_{2}$ 与溶液中的 $\mathrm{Ca}^{2+}$ 之间的反应, 可以利用气液界面胶体球 刻蚀法获得大面积有序的无定形碳酸钙的纳米碗阵列 薄膜, 它还可以进一步晶化成为方解石碳酸钙的蜂窝状 薄膜 ${ }^{[25]}$.

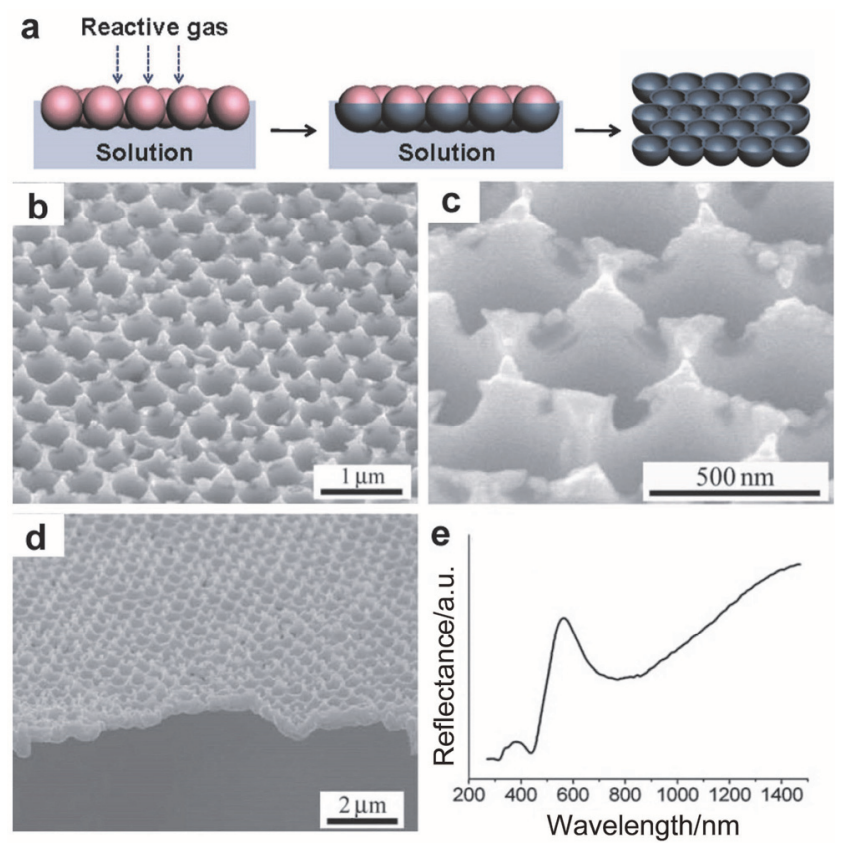

图 4 (a) 基于气体扩散的气液界面胶体球刻蚀法制备纳米碗阵列示 意图; (b $\sim \mathrm{d}) \mathrm{Ag}$ 纳米碗阵列的 $\mathrm{SEM}$ 照片; (e) $\mathrm{Ag}$ 纳米碗阵列的反射光 谱[24]

Figure 4 (a) Schematic of the fabrication of nanobowl arrays by nanosphere lithography at the gas-liquid interface via gas diffusion. $(b \sim d)$ SEM images of Ag nanobowl arrays. (e) Reflection spectrum of $\mathrm{Ag}$ nanobowl arrays ${ }^{[24]}$
上述基于气体扩散反应的气液界面胶体球刻蚀法 在制备易于沉积的无机物种时，能够取得好的效果. 对 于溶度积较高的物种, 则需要做适当调整. 我们在制备 $\mathrm{ZnS}$ 纳米网阵列时, 便采用了基于直接液相沉积的气液 界面胶体球刻蚀法(图 5) ${ }^{[26]}$. 将 $\mathrm{MCC}$ 转移至 $\mathrm{Zn}\left(\mathrm{CH}_{3} \mathrm{COO}\right)_{2} 、 \mathrm{TAA} 、 \mathrm{Na}_{2} \mathrm{EDTA} 、 \mathrm{CH}_{3} \mathrm{COONH}_{4}$ 的混合 溶液表面, 利用 TAA 在溶液中逐渐释放出的 $\mathrm{S}^{2-}$ 与 $\mathrm{Zn}^{2+}$ 发生反应, 即可在胶球的底部原位生成 $\mathrm{ZnS}$ 纳米碗(图 5a). 这里 $\mathrm{Na}_{2}$ EDTA 和 $\mathrm{CH}_{3} \mathrm{COONH}_{4}$ 的加入有利于提高 纳米碗阵列的均一性及规整性. 若以 $600 \mathrm{~nm}$ 的胶球为 模板, 则可得到高度为 $350 \mathrm{~nm}$, 厚度约为 $80 \mathrm{~nm}$ 的大面 积连续的 $\mathrm{ZnS}$ 纳米碗阵列(图 $5 \mathrm{~b} \sim 5 \mathrm{e}$ ). 图 $5 \mathrm{~d}$ 给出了纳

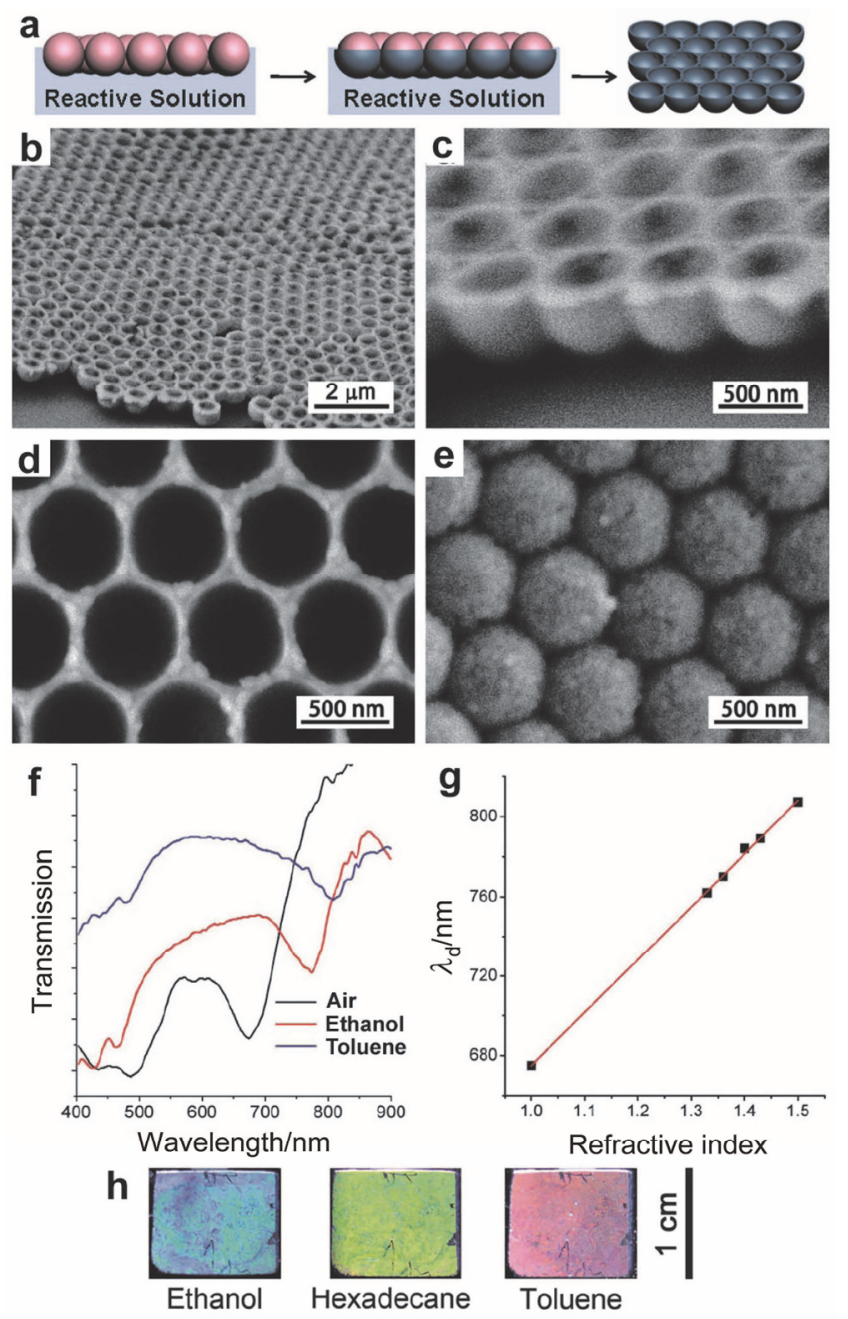

图 5 (a) 基于液相沉积的气液界面胶体球刻蚀法制备纳米碗阵列示 意图; $(\mathrm{b} \sim \mathrm{e}) \mathrm{ZnS}$ 纳米碗阵列的 SEM 照片; (f) 不同溶剂中 $\mathrm{ZnS}$ 纳米碗 阵列的透射光谱; (g) 透射波谷随溶剂折光指数的变化; (h) 不同溶剂 中 $\mathrm{ZnS}$ 纳米碗阵列的光学照片 ${ }^{[26]}$

Figure 5 (a) Schematic of the fabrication of nanobowl arrays by nanosphere lithography at the gas-liquid interface via solution deposition. (b e) SEM images of $\mathrm{ZnS}$ nanobowl arrays. (f) Transmission spectra of $\mathrm{ZnS}$ nanobowl arrays immersed in different organic solvents. (g) Relationship between the stop band position and the refractive index of solvents. (h) Optical photographs of $\mathrm{ZnS}$ nanobowl arrays immersed in three different solvents $^{[26]}$ 
米碗阵列背面的 SEM 照片, 它显示出纳米碗具有封闭 的碗底, 且是由 $\mathrm{ZnS}$ 纳米粒子堆积而成.

$\mathrm{ZnS}$ 是一种高折射率 $(n \approx 2.35)$ 的宽带隙半导体材 料, 因而在构筑基于可见及近红外光的光子晶体方面具 有独特的优势. $\mathrm{ZnS}$ 纳米碗阵列是一种典型的二维光子 晶体, 其光子带隙与周围介质的折射率密切相关. 图 $5 \mathrm{f}$ 给出了浸泡于不同有机溶剂中的 $\mathrm{ZnS}$ 纳米碗阵列的透 射光谱. 随着空气、乙醇、甲苯的折射率的依次增大, 透 射波谷发生明显的红移. 其透射波谷或光子带隙与溶剂 的折射率之间存在良好的线性关系(图 $5 \mathrm{~g}$ ), 检测灵敏度 高达 $266 \mathrm{~nm} / \mathrm{RIU}$. 此外, 该纳米碗阵列还可通过宏观颜 色的变化实现对不同折射率的有机溶剂的可视化检测. 当 $\mathrm{ZnS}$ 纳米碗阵列浸泡在乙醇、十六烷、甲苯中时, 它 的颜色相应地变为蓝色、黄色与红色(图 5h). 因此, 该 $\mathrm{ZnS}$ 纳米碗阵列可以被用作灵敏度高、快速及可视化的 二维光子晶体传感器.

若将生物素 (biotin) 标记的牛血清白蛋白 (biotin-LC-BSA)修饰到 $\mathrm{ZnS}$ 纳米碗阵列基底上, 结合生 物素与抗生物素蛋白(avidin)的特异性识别, 还可实现 对抗生物素蛋白的快速定量检测(图 6) ${ }^{[26]}$. 图 6a 给出了 $\mathrm{ZnS}$ 纳米碗阵列用作二维光子晶体生物传感器的工作原 理示意图. 首先, 将 biotin-LC-BSA 吸附在 $\mathrm{ZnS}$ 纳米碗 阵列表面; 然后, 利用生物素及抗生物素蛋白之间的特 异性相互作用, 可以利用生物素修饰的 $\mathrm{ZnS}$ 纳米碗阵列 对溶液中的抗生物素蛋白进行检测. biotin-LC-BSA 的 吸附使 $\mathrm{ZnS}$ 纳米碗附近的折射率变大, 因此其透射波谷 由原始 $\mathrm{ZnS}$ 纳米碗的 $672 \mathrm{~nm}$ 移动至 $674 \mathrm{~nm}$; 将修饰了 生物素的纳米碗阵列浸入抗生物素蛋白溶液中一段时 间, 透射波谷进一步红移至 $682 \mathrm{~nm}$ (图 6b). 当抗生物素 蛋白溶液浓度较低时(小于 $500 \mathrm{pmol} / \mathrm{L}$ ), 抗生物素蛋白 浓度与碗阵列透射光谱带隙波长变化值存在线性关系,
检出限低于 $100 \mathrm{pmol} / \mathrm{L}$ (图 6c). 若抗生物素蛋白浓度较 高，则抗生物素蛋白浓度与光子带隙波长变化之间的关 系不再线性(图 6d). 除了具有超低的检出限、检测范围 宽 $(100 \mathrm{pmol} / \mathrm{L} \sim 100 \mathrm{nmol} / \mathrm{L})$ 的特点外, 该阵列的开放式 碗状结构有利于检测分子的快速吸附.

\section{5 纳米网-纳米碗复合阵列}

由窄带隙半导体 $\mathrm{Ag}_{2} \mathrm{~S}$ 与贵金属复合而成的纳米异 质结构受到人们的广泛关注, 然而目前所报道的 $\mathrm{Ag}_{2} \mathrm{~S}$ 贵金属纳米异质结构大多为彼此分离的单个纳米结构. 因此，发展一种简便易行的制备半导体一贵金属纳米异 质结构的大面积有序阵列的合成策略是人们面临的一 项挑战. 基于上述制备自支撑 $\mathrm{Ag}_{2} \mathrm{~S}$ 纳米网和 $\mathrm{Ag}$ 纳米碗 阵列的研究基础, 最近我们通过基于两步化学沉积的气 液界面胶体球刻蚀法成功实现了 $\mathrm{Ag}_{2} \mathrm{~S}$ 纳米网 $-\mathrm{Ag}$ 纳米 碗复合阵列的可控制备(图 7) ${ }^{[27]}$. 如图 7a 所示, 以漂浮 在 $\mathrm{AgNO}_{3}$ 溶液表面的 $\mathrm{MCC}$ 作为模板, 在体系中先引入 $\mathrm{H}_{2} \mathrm{~S}$ 气体生成 $\mathrm{Ag}_{2} \mathrm{~S}$ 纳米网, 再引入 $\mathrm{N}_{2} \mathrm{H}_{4} / \mathrm{NH}_{3}$ 混合气体 生成附着于 $\mathrm{Ag}_{2} \mathrm{~S}$ 纳米网下面的 $\mathrm{Ag}$ 纳米碗阵列, 从而制 备得到了大面积连续的 $\mathrm{Ag}_{2} \mathrm{~S}-\mathrm{Ag}$ 异质结构纳米碗阵列 (面积可达 $2 \times 2 \mathrm{~cm}^{2}$ ). SEM 照片清楚显示了该异质结构 纳米碗阵列的形貌构造(图 $7 \mathrm{~b} \sim 7 \mathrm{~d}$ ). 能谱分析(EDS)结 果表明 $\mathrm{Ag}$ 元素在整个结构中都存在, 而 $\mathrm{S}$ 元素仅存在 于 $\mathrm{Ag}_{2} \mathrm{~S}$ 网的位置, 从而证实了 $\mathrm{Ag}_{2} \mathrm{~S}-\mathrm{Ag}$ 异质结构的生 成 (图 7e). 与单纯的 $\mathrm{Ag}_{2} \mathrm{~S}$ 纳米网及 $\mathrm{Ag}$ 纳米碗阵列相比, 两步法制备得到的复合材料均保持了两种结构的原有 规整形貌，这也进一步证明纳米网和纳米碗的成核位置 相互独立，因而原理上也可适用于其他物质复合结构的 制备.

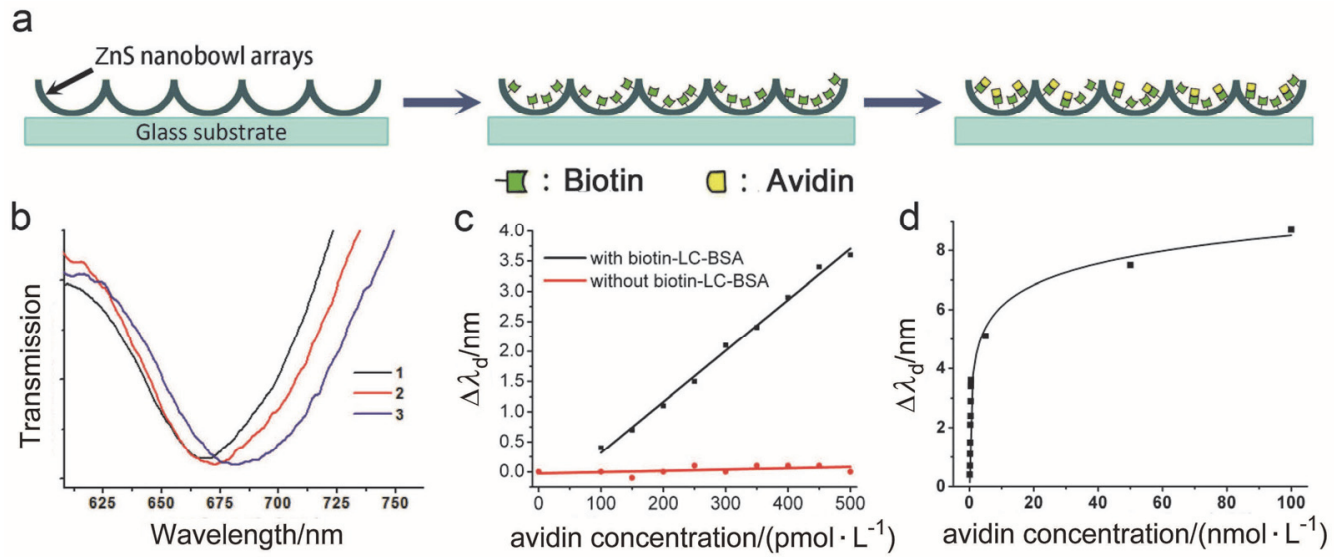

图 6 (a) 基于 $\mathrm{ZnS}$ 纳米碗阵列检测抗生物素蛋白的示意图; (b) 吸附了不同分子的 $\mathrm{ZnS}$ 纳米碗阵列的透射光谱: (1) 未吸附分子, (2) 已吸附生物 素分子, (3) 已捕获抗生物素蛋白; (c, d) 透射波谷改变值随抗生物素蛋白浓度的变化曲线 ${ }^{[26]}$

Figure 6 (a) Scheme illustration of the procedure for the operation of avidin molecule detection based on ZnS nanobowl arrays. (b) Transmission spectra of $\mathrm{ZnS}$ nanobowl arrays with different adsorbed molecules: (1) as-synthesized without adsorbents, (2) biotin-LC-BSA-adsorbed, (3) avidin-captured (100 $\mathrm{nmol} / \mathrm{L}$ ). (c, d) Relationship between the change of the stop band position and the avidin concentration in the concentration range of $0 \sim 500 \mathrm{pmol} / \mathrm{L}$ (c) and $0 \sim 100 \mathrm{nmol} / \mathrm{L}$ (d). For comparison purposes, the situation of the $\mathrm{ZnS}$ nanobowl arrays without adsorption of biotin-LC-BSA is also shown in (c) ${ }^{[26]}$ 


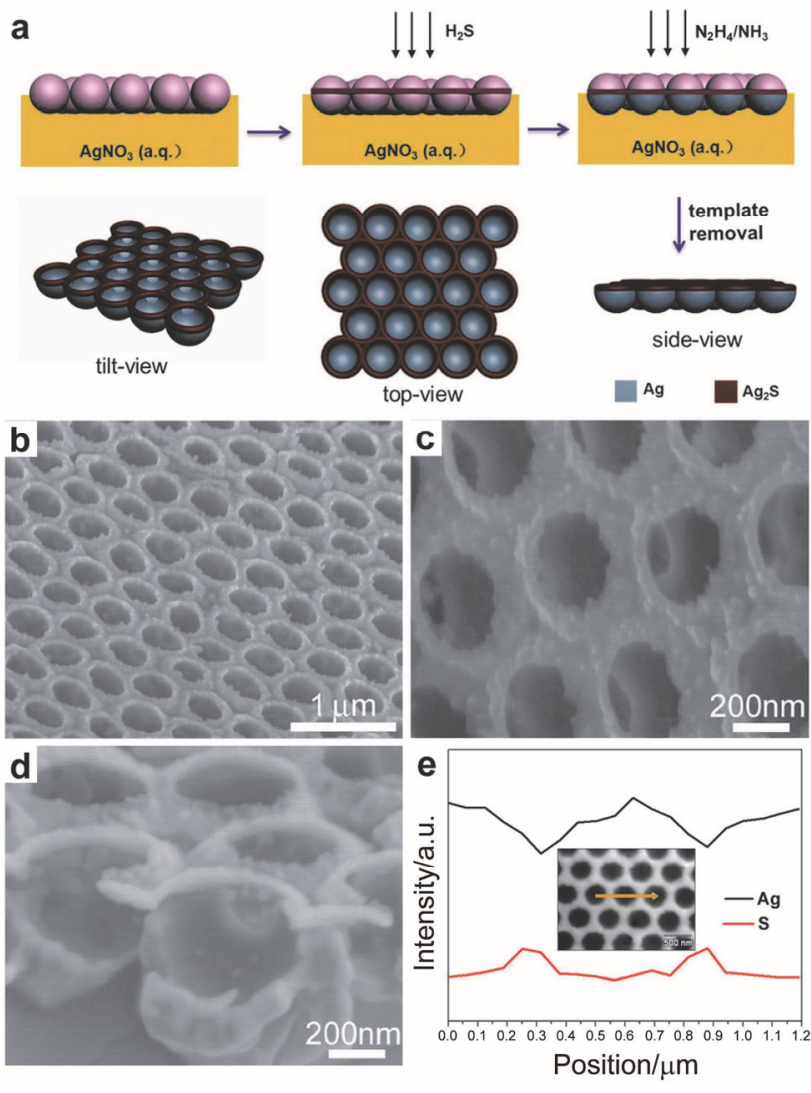

图 7 (a) 两步气液界面胶体球刻蚀法制备 $\mathrm{Ag}_{2} \mathrm{~S}-\mathrm{Ag}$ 异质结构纳米碗 阵列示意图; (b d) $\mathrm{Ag}_{2} \mathrm{~S}-\mathrm{Ag}$ 异质结构纳米碗阵列的 SEM 照片; (e) EDS 线扫元素分布曲线 ${ }^{[27]}$

Figure 7 (a) Schematic of the fabrication of $\mathrm{Ag}_{2} \mathrm{~S}-\mathrm{Ag}$ heterostructured nanobowl arrays by two-step nanosphere lithography at the gas-liquid interface. ( $b \sim d)$ SEM images of $\mathrm{Ag}_{2} \mathrm{~S}-\mathrm{Ag}$ heterostructured nanobowl arrays. (e) Elemental profile of line scanning by EDS analysis ${ }^{[27]}$

所制备得到的 $\mathrm{Ag}_{2} \mathrm{~S}-\mathrm{Ag}$ 异质结构纳米碗阵列可以 很方便地转移至氟掺杂氧化锡(FTO)透明导电玻璃表面, 并分别制备成具有典型电阻开关效应的 $\mathrm{FTO} / \mathrm{Ag}_{2} \mathrm{~S} / \mathrm{Ag} / \mathrm{FTO}$ 电学器件和具有显著光响应特性的 $\mathrm{FTO} / \mathrm{Ag} / \mathrm{Ag}_{2} \mathrm{~S}$ 光阳极 (图 8$)^{[27]}$. 图 $8 \mathrm{a}$ 给出了 $\mathrm{FTO} / \mathrm{Ag} / \mathrm{Ag}_{2} \mathrm{~S} / \mathrm{FTO}$ 电阻开关器件的结构示意图. 图 $8 \mathrm{~b}$ 显示, 在进行循环伏安扫描时, 电流 $(I)$ 随外加电压 $(V)$ 的 变化会发生周期性的突变, 呈现出典型的电阻开关效 应, 最大电阻开关比约为 54. 这种电阻开关效应可归因 于外加电压变化时 $\mathrm{Ag}_{2} \mathrm{~S}$ 内部 $\mathrm{Ag}$ 线的生成和溶解所分 别导致的 $\mathrm{Ag}_{2} \mathrm{~S}-\mathrm{Ag}$ 复合结构电阻的减小和增大 ${ }^{[28]}$. 若将 转移至 $\mathrm{FTO}$ 基底上的 $\mathrm{Ag}_{2} \mathrm{~S}-\mathrm{Ag}$ 异质结构纳米碗阵列作 为光电化学池的光阳极, 可以呈现出显著增强的光电化 学分解水性能(图 8c). 一方面 $\mathrm{Ag}$ 纳米碗层的存在有利 于光生电子由 $\mathrm{Ag}_{2} \mathrm{~S}$ 向 $\mathrm{Pt}$ 电极的传递及由此带来的电荷 分离, 另一方面, $\mathrm{Ag}$ 纳米碗有利于光线在其内部的多次 散射, 从而有利于可见光的捕获利用. 这两方面因素均 有利于其光响应性能的增强. 如图 $8 \mathrm{~d}$ 所示, 在外加电压 为 $1.23 \mathrm{~V}$ 时, $\mathrm{Ag}_{2} \mathrm{~S}-\mathrm{Ag}$ 异质结构纳米碗阵列的光电流密
度为 $17.9 \mu \mathrm{A} \cdot \mathrm{cm}^{-2}$, 较之单纯 $\mathrm{Ag}_{2} \mathrm{~S}$ 纳米网的光电流密 度 $\left(2.1 \mu \mathrm{A} \cdot \mathrm{cm}^{-2}\right)$ 有很大提高, 表现出显著增强的光催化 活性. 该结果揭示了气液界面胶体球刻蚀法在制备具有 多重功能的半导体-贵金属异质结构纳米阵列方面的应 用潜力.

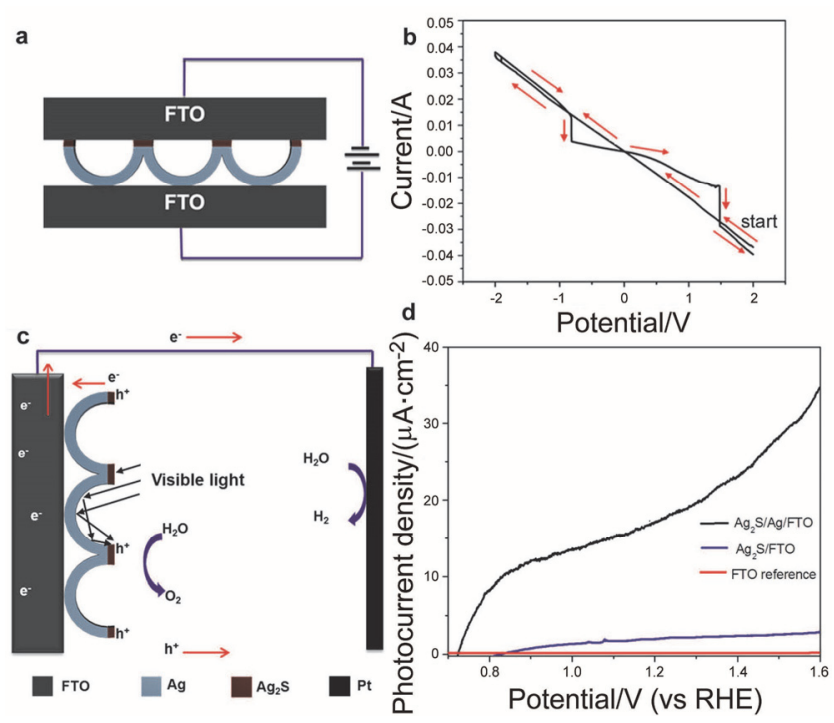

图 $8 \mathrm{FTO} / \mathrm{Ag} / \mathrm{Ag}_{2} \mathrm{~S} / \mathrm{FTO}$ 电阻开关器件的结构示意图(a)和 $I-V$ 曲线 (b); (c) 以 $\mathrm{Ag}_{2} \mathrm{~S} / \mathrm{Ag} / \mathrm{FTO}$ 为光阳极的光电化学池工作原理图; (d) 可见光 照射下不同光阳极的线扫伏安曲线 ${ }^{[27]}$

Figure 8 Schematic structure (a) and $I-V$ curve (b) of $\mathrm{FTO} / \mathrm{Ag} / \mathrm{Ag}_{2} \mathrm{~S} / \mathrm{FTO}$ device made of the heterostructured nanobowl arrays. (c) Schematic diagram of electron/hole transport for water splitting in an $\mathrm{Ag}_{2} \mathrm{~S} / \mathrm{Ag} / \mathrm{FTO}$ photoelectrochemcial cell (PEC) using the $\mathrm{Ag}_{2} \mathrm{~S} / \mathrm{Ag} / \mathrm{FTO}$ as the photoanode. (d) Linear sweep voltammograms curves under visible light illumination for $\mathrm{Ag}_{2} \mathrm{~S} / \mathrm{Ag} / \mathrm{FTO}$ and $\mathrm{Ag}_{2} \mathrm{~S} / \mathrm{FTO}$ PECs together with blank $\mathrm{FTO}^{[27]}$

\section{6 总结与展望}

气液界面胶体球刻蚀法的发展为二维有序多孔薄 膜的可控制备提供了一条新的途径，它具有装置简单、 操作方便、可重复性好、适用范围广、结构参数易调变、 适于制备大面积自支撑薄膜等优点，因而具有广阔的发 展前景. 我们近期的研究工作表明, 气液界面胶体球刻 蚀法有望成为制备无机纳米网、纳米碗阵列以及纳米 网一纳米碗复合阵列的一种通用合成方法. 我们已利用 该方法成功制备得到大面积规整有序的 $\mathrm{Ag}_{2} \mathrm{~S}$ 纳米网, $\mathrm{Ag} 、 \mathrm{ZnS} 、 \mathrm{CaCO}_{3}$ 纳米碗阵列, $\mathrm{Ag}_{2} \mathrm{~S}-\mathrm{Ag}$ 异质结构纳米碗 阵列等多种自支撑二维有序多孔薄膜, 并研究了其光谱 性质及在刻蚀掩膜、溶剂检测、生物传感、电阻开关器 件、光电化学分解水等方面的应用. 值得指出的是, 这 一领域近期受到愈来愈多的关注. 例如, 在聚合物多孔 薄膜方面, Fujii 等 ${ }^{[29]}$ 采用该方式制备了一种由聚吡咯纳 米碗阵列构成的柔性 Janus 型胶体晶体薄膜, 在微反应 器等领域具有潜在的应用价值; Goedel 等 ${ }^{[30]}$ 则利用由两 种不同尺寸胶球构成的二元胶体晶体作为模板, 制备了 
多级有序的、含有微篮结构的不对称型聚合物多孔薄膜; Kamperman 等 ${ }^{[31]}$ 利用气液界面胶体球刻蚀法制备了具 有显著增强粘附性能的聚二甲基硅氧烷(PDMS)纳米碗 阵列, 可以作为具有仿生特性的高粘性材料使用. 在无 机多孔薄膜方面, 通过对气液界面胶体球刻蚀法制备的 $\mathrm{ZnS}$ 纳米碗阵列进行热处理, 可以实现对其形貌和发光 性能的调节 ${ }^{[32]}$; 结合光诱导的方法制备出可直接用于 电阻型气体传感器的 $\mathrm{SnO}_{2}$ 二维有序多孔薄膜 ${ }^{[33]}$; 利用 气液界面胶体球刻蚀法制备出可用于电致变色器件的 大面积无缺陷 $\mathrm{WO}_{3}$ 二维有序多孔薄膜 ${ }^{[34]}$; 还利用该方 法制备出可用于光致变色器件的 $\mathrm{TiO}_{2}-\mathrm{WO}_{3}$ 复合二维有 序多孔薄膜 ${ }^{[35]}$.

有关气液界面胶体球刻蚀法的研究近年来取得了 显著进展, 但整体上仍处于初始阶段, 还有很大的发展 空间. 以下为该领域值得关注的一些研究方向: (1) 单 层胶体晶体模板的优化与调控. 首先, 探索大面积、高 质量单层胶体晶体的简便易行、高重现性、低成本的制 备方法仍是一个值得深入研究的课题, 它将有助于复制 得到价格低廉的高质量二维有序多孔薄膜. 其次, 对单 层胶体晶体的结构参数进行适当的人为调控将有助于 获得具有新颖结构的二维有序多孔薄膜. 例如, 对气液 界面处的胶球进行原位加热可方便地控制胶球之间的 缝隙 ${ }^{[36]}$, 而借助于聚合物胶球溶胀与溶解行为可以制 备 Janus 型半球胶体晶体薄膜 ${ }^{[37]}$. 此外, 通过在气液界 面组装结构多样的二元单层胶体晶体 ${ }^{[38,39]}$, 有望复制得 到形态复杂多样的二维有序多孔薄膜, 带来更多的独特 性质或优化性能. (2) 模板复制方法的优化和材料体系 的拓展. 目前已经通过气液界面胶体球刻蚀法实现了包 括聚合物、金属、硫化物、氧化物及难溶盐在内的多种 物质二维有序多孔薄膜的可控制备, 但其中有的合成产 物的整体质量有待提高 ${ }^{[40]}$, 需要在深入研究模板复制 机理的基础上进一步优化合成方法; 应继续探索一些新 的模板复制方法, 以便适用于其它各种功能性材料体 系, 并实现随意选择得到纳米网或纳米碗阵列结构; 此 外, 还应继续探索具有特定功能或多重功能的复合二维 有序多孔薄膜的制备方法. (3) 二维有序多孔薄膜的性 能研究与应用. 应深入研究二维有序多孔薄膜的结构与 性能之间的关系, 并充分发挥出其二维有序多孔结构在 应用方面的优势. 应不断发掘二维有序多孔结构可能所 带来的新奇性质或优化性能, 为气液界面胶体球刻蚀法 的发展提供新的应用导向. 例如, 近期人们发现有序多 孔薄膜构成的超颖表面(metasurface)已显示出许多奇特 性质和广阔应用前景 ${ }^{[4]}$. 此外, 还应注重解决利用气液 界面胶体球刻蚀法制备的二维有序多孔薄膜在走向应 用过程中可能遇到的一些实际问题, 积极推动其在各个 重要技术领域的实际应用.

\section{作者简介}

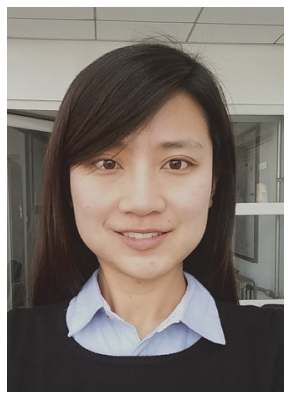

李扬, 北京大学化学与分子工程学院 2011 级硕博连读研 究生. 2011 年获天津大学理学学士学位. 研究方向为基于单层 胶体晶体模板的纳米材料合成与性能研究.

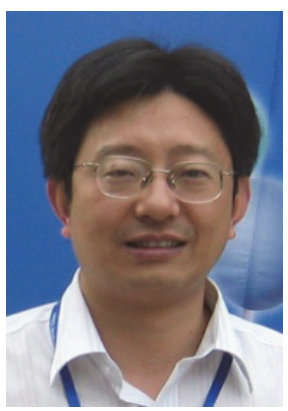

齐利民, 北京大学化学与分子工程学院教授, 国家杰出 青年基金获得者. 主要从事胶体与界面化学、纳米材料、能源 材料和仿生材料等领域的研究. 迄今发表学术论文 150 余篇, 累计被引用逾 8800 次, $\mathrm{H}$ 因子为 54 .

\section{References}

[1] Ye, X.; Qi, L. Nano Today 2011, 6, 608.

[2] Warkiani, M. E.; Bhagat, A. A. S.; Khoo, B. L.; Han, J.; Lim, C. T.; Gong, H. Q.; Fane, A. G. ACS Nano 2013, 7, 1882.

[3] van Rijn, P.; Tutus, M.; Kathrein, C.; Zhu, L.; Wessling, M.; Schwaneberg, U.; Böker, A. Chem. Soc. Rev. 2013, 42, 6578.

[4] Yu, N; Capasso, F. Nat. Mater. 2014, 13, 139.

[5] Ji, L.; McDaniel, M. D.; Wang, S.; Posadas, A. B.; Li, X.; Huang, H.; Lee, J. C.; Demkov, A. A.; Bard, A. J.; Ekerdt, J. G.; Yu, E. T. Nat. Nanotechnol. 2015, 10, 84 .

[6] Zhou, L.; Zhang, L.; Liao, L.; Yang, M.; Xie, Q.; Peng, H.; Liu, Z.; Liu, Z. Acta Chim. Sinica 2014, 72, 289. (周琳，张黎明，廖否，杨 明媚, 谢芹, 彭海琳, 刘志荣, 刘忠范, 化学学报, 2014, 72, 289.)

[7] Lei, Y.; Yang, S.; Wu, M.; Wilde, G. Chem. Soc. Rev. 2011, 40, 1247.

[8] Li, Y.; Cai, W.; Duan, G. Chem. Mater. 2007, 20, 615.

[9] Zhang, J.; Li, Y.; Zhang, X.; Yang, B. Adv. Mater. 2010, 22, 4249.

[10] Zhang, G.; Zhao, Z.-Y.; Wang, D.-Y. Chem. J. Chin. Univ. 2010, 31, 839. (张刚, 赵志远, 汪大洋, 高等学校化学学报, 2010, 31, 839.)

[11] Li, Y.; Duan, G.; Liu, G.; Cai, W. Chem. Soc. Rev. 2013, 42, 3614.

[12] Geng, C.; Wei, T.; Wang, X.; Shen, D.; Hao, Z.; Yan, Q. Small 2014, $10,1668$.

[13] Ye, X.; Qi, L. Sci. China Chem. 2014, 57, 58.

[14] Ma, H.; Hao, J. Chem. Soc. Rev. 2011, 40, 5457.

[15] Bai, H.; Du, C.; Zhang, A.; Li, L. Angew. Chem. Int. Ed. 2013, 52, 12240.

[16] Xu, H.; Goedel, W. A. Angew. Chem. Int. Ed. 2003, 42, 4694.

[17] Yan, F.; Goedel, W. A. Chem. Mater. 2004, 16, 1622.

[18] Yan, F.; Goedel, W. A. Nano Lett. 2004, 4, 1193.

[19] Xu, H.; Goedel, W. A. Angew. Chem. Int. Ed. 2003, 42, 4696.

[20] Chen, J.; Chao, D.; Lu, X.; Zhang, W.; Manohar, S. K. Macromol. Rapid Commun. 2006, 27, 771 .

[21] Jiang, S.; Chen, J.; Tang, J.; Jin, E.; Kong, L.; Zhang, W.; Wang, C. 
Sens. Actuators, B-Chem. 2009, 140, 520

[22] Sun, F. Q.; Yu, J. C. Angew. Chem. Int. Ed. 2007, 46, 773.

[23] Li, C.; Hong, G.; Qi, L. Chem. Mater. 2010, 22, 476.

[24] Hong, G.; Li, C.; Qi, L. Adv. Funct. Mater. 2010, $20,3774$.

[25] Li, C.; Hong, G.; Yu, H.; Qi, L. Chem. Mater. 2010, 22, 3206.

[26] Ye, X.; Li, Y.; Dong, J.; Xiao, J.; Ma, Y.; Qi, L. J. Mater. Chem. C 2013, 1,6112

[27] Li, Y.; Ye, X.; Ma, Y.; Qi, L. Small 2015, 11, 1183.

[28] Wang, H.; Qi, L. Adv. Funct. Mater. 2008, 18, 1249.

[29] Fujii, S.; Kappl, M.; Butt, H. J.; Sugimoto, T.; Nakamura, Y. Angew. Chem. Int. Ed. 2012, 51, 9809.

[30] Wachner, D.; Marczewski, D.; Goedel, W. A. Adv. Mater. 2013, 25, 278.

[31] Akerboom, S.; Appel, J.; Labonte, D.; Federle, W.; Sprakel, J.; Kamperman, M. J. R. Soc. Interface 2015, 12, 20141061.
[32] Liu, Y.; Li, Z.; Zhong, W.; Zhang, L.; Chen, W.; Li, Q. Nano Res. Lett. 2014, 9, 389.

[33] Xu, S.; Sun, F.; Gu, F.; Zuo, Y.; Zhang, L.; Fan, C.; Yang, S.; Li, W. ACS Appl. Mater. Interfaces 2014, 6, 1251.

[34] Li, H.; Thériault, J.; Rousselle, B.; Subramanian, B.; Robichaud, J.; Djaoued, Y. Chem. Commun. 2014, 50, 2184.

[35] Zhen, H.; Li, K. New J. Chem. 2014, 38, 4041.

[36] Geng, C.; Zheng, L.; Yu, J.; Yan, Q.; Wei, T.; Wang, X.; Shen, D. J. Mater. Chem. 2012, 22, 22678.

[37] Zhang, J. T.; Chao, X.; Asher, S. A. J. Am. Chem. Soc. 2013, 135, 11397.

[38] Yu, J.; Yan, Q.; Shen, D. ACS Appl. Mater. Interfaces 2010, $2,1922$.

[39] Dai, Z.; Li, Y.; Duan, G.; Jia, L.; Cai, W. ACS Nano 2012, 6, 6706.

[40] Guo, Y.; Yang, M.; Wu, Q. Acta Chim. Sinica 2013, 71, 693. (郭阳 光, 杨穆, 吴强, 化学学报, 2013, 71, 693.) 